\title{
PhD Thesis Writing Process: A Systematic Approach-How to Write Your Introduction
}

\author{
Qais Faryadi \\ Future Expert Solutions, Creative Research and Innovations, Kuala Lumpur, Malaysia \\ Email: user@magnesium4you.com
}

How to cite this paper: Faryadi, Q. (2018). PhD Thesis Writing Process: A Systematic Approach-How to Write Your Introduction. Creative Education, 9, 2534-2545. https://doi.org/10.4236/ce.2018.915192

Received: October 23, 2018

Accepted: November 17, 2018

Published: November 20, 2018

Copyright $\odot 2018$ by author and Scientific Research Publishing Inc. This work is licensed under the Creative Commons Attribution International License (CC BY 4.0).

http://creativecommons.org/licenses/by/4.0/

\begin{abstract}
Thesis writing is a skill that every $\mathrm{PhD}$ candidate must acquire to convey his or her research findings clearly. The main objective of this paper is to facilitate the thesis writing process so that $\mathrm{PhD}$ candidates understand what a $\mathrm{PhD}$ thesis is and can write their thesis correctly and scientifically. The methodology used in this research was descriptive as it discusses and describes the various parts of thesis writing process and explains how to do it in a very simple and understanding language. As thus, this article outlines the various steps of thesis writing to guide the $\mathrm{PhD}$ candidate so that the task of $\mathrm{PhD}$ thesis writing becomes manageable and less daunting. This research is a useful roadmap especially for students of the social sciences studies. Further, in this paper, research procedure and thesis writing strategies are explained in a simple manner. This paper adopts a how-to approach when discussing a variety of relevant topics, such as thesis introduction, types of introductions, introduction statements, problem statement, research questions, hypothesis and contributions of the study. This paper has 5 parts: Introduction, Literature Review, Methodology, Results and Conclusion. The introduction chapter is discussed in this paper. I will discuss the rest as a series in the future.
\end{abstract}

\section{Keywords}

Thesis Writing Process, Learning and Teaching, PhD, Social Science, Research Methodology, Introduction

\section{Introduction}

Before you write your introduction, you must know what a research is first. It makes every stage of writing easier. Research is defined as a premeditated investigation using scientific methodology (quantitative, qualitative, experimental, observation and so on) to solve a serious problem (not ordinary problem), thus 
creating additional (new) knowledge. Research is also regarded as an inquiry of reality about something by testing a hypothesis, answering questions, generating new queries, finding solutions, and creating new knowledge. The new knowledge must be applicable/defensible and challengeable by other researchers, besides lending itself to generalization. For a piece of exploration to qualify as a research, it must pass through a set of rigorous examinations, such as validity (logical procedure to answer a question), reliability (quality of measurement), and unbiased conclusion (adequate measures are taken to make sure that it is free from individual interest). As such, a $\mathrm{PhD}$ thesis must meet the above conditions of a scientific research procedure (Mincu, 2015).

\section{Problem Statement}

Thesis writing is a skill that every $\mathrm{PhD}$ candidate must acquire to convey his or her research findings clearly. Unfortunately majority of $\mathrm{PhD}$ candidates find it difficult to finish their thesis on time because of confusion and lake of expertise. Most of them in deed do not know how to write the PhD thesis correctly and scientifically.

\section{Objectives}

1) To help $\mathrm{PhD}$ candidates in writing scientifically correct $\mathrm{PhD}$ thesis.

2) To describe $\mathrm{PhD}$ thesis writing process.

3) To assist $\mathrm{PhD}$ candidates to understand what $\mathrm{PhD}$ means.

\section{Methodology}

The methodology applied in this research was descriptive as it discusses and describes the various parts of $\mathrm{PhD}$ thesis and explains the how to do of them in a very simple and understanding language. Descriptive analysis is applied to explain the basic features of thesis writing process (García \& Fombona, 2015). Descriptive method is very useful in providing basic summaries of the chapters (Al-Raqqad et al., 2017). The following are the process of $\mathrm{PhD}$ thesis writing process.

\subsection{Introduction Writing Process}

An introduction is the most difficult parts of a $\mathrm{PhD}$ thesis. The introduction opens a dialogue with your examiner or reader. As such, a good introduction is critical to capturing the attention of your readers and engaging their interest in your research. Your introduction must convince your reader that you are the right person among thousands of researchers. You must also show your reader how you going to fulfil their needs and what exceptional benefits they can get from you as a researcher. You should convince your readers that you are an authority on the subject of investigation (Faryadi, 2017). Consult your supervisors or your department about the format of your thesis before starting to write. Institutions may vary in their requirements with regard to thesis-writing. Never- 
theless, the following checklist provides some general guidelines:

1) Consult your supervisors.

2) Familiarize yourself with the style or format of thesis writing.

3) Decide on the word length. How many words your thesis should be.

4) Learn more about referencing styles.

5) Make sure you have all the necessary information for the reference section.

6) Learn about indexing of your thesis.

\subsection{Tips for Writing the Introduction}

1) State the problem or phenomenon to be investigated.

2) Identify the party affected by the problem.

3) Explain how you plan to solve the problem.

4) Convince the reader that you are qualified and equipped with the right methods of solving that problem.

5) Highlight the benefits of solving the problem.

6) Tell the reader what results you anticipate.

a) As evident from the above, in your introduction, you should communicate the rationale of your research. Explain the importance of your research. Even though your research introduction chapter has no word limit, being concise helps your readers to comprehend quickly the major issues in your research. When you eventually start writing the introduction, start with some relevant general statements before gradually narrowing it down to focus on the crucial issues such as your research problem, questions, objectives and hypothesis. Here, you explain briefly your research problem and how you plan to solve it. Your introduction must be motivating and captivating enough so that the reader will want to read on to find out more.

b) It is assumed that your introduction is prepared for readers who have adequate knowledge of your discipline. Your introduction includes many crucial aspects of your thesis. Readers may want to know whether it is your own discovery, or you are continuing a previous study. If it is your own scientific discovery, then you must make it clear how the findings will add to the existing knowledge (Kafes, 2018). Your reader would also want to know more about the concepts used in your research, the objectives of the research, and the methodology employed. You could also discuss the obstacles encountered, if any, and the limitation of your research.

\subsection{Roadmap for Developing Your Introduction}

1) Write clearly.

2) Be precise and concise in your introduction.

3) State your problem statement clearly and explain why the problem is worthy of investigation.

4) State your objectives clearly.

5) State your hypothesis and explain briefly how you would test it. 
6) Keep the best for the last. Your introduction is one of the best parts of your thesis. If it is possible, write it once everything is finished. In this manner, you will not miss anything important and significant.

As you can see from the above guidelines, the introduction is crucial in conveying your ideas, thoughts and your message to the world. A good introduction paves the way to showcasing your thesis. As such, while it is important to convey your message loud and clear, your introduction must be well-organized, convincing, and be engaging. Prepare your introduction as a preview to your readers so that they can judge the rest of your work and its significance at a glance (BCCC Tutoring Centre).

\subsection{Type of Introductions}

1) Illusion Introduction: Start your introduction with an illusion style statement so that your reader cannot resist reading on.

2) Analogue Introduction: Start your introduction with a comparison so that your reader is eager to know the differences.

3) Narrative Introduction: Start your introduction with an eye-catching story to capture your reader's attention.

4) Descriptive introduction: Start your introduction with a descriptive statement about your title. Once you start describing something, readers become eager to continue reading.

5) Definition Introduction: You may begin your introduction by defining a part of your topic, thus creating a knowledge transferring environment for your reader.

6) Example Introduction: Begin your introduction with an interesting example so that your reader takes it as personal motivation for writing his/her own thesis introduction.

7) Question Introduction: Start your introduction by posing a question; it will keep your reader thinking and yearning to know the answer.

8) Statistic Introduction: You can start your introduction with statistics concerning the parties affected by the problem that you will investigate.

9) Quotation Introduction: Start your introduction with a quotation that is relevant to $\mathrm{PhD}$ thesis writing so that your reader takes it as a personal motivation.

\subsection{What Is an Introduction Statement?}

Your thesis statement is a declaration of your topic and a claim of how you are going to prove or disprove your problem. It is found in the first paragraph of the introduction where you declare that you have an important subject to present. Your argument should be persuasive or informative. In any of these two methods of presentation, you show your assertion and view point about your entire thesis to your readers (Koçyigit \& Erdem, 2018). Here is how you write a good thesis statement: 
1) Make an assertion about your style of interpretation.

2) Capture the attention of your reader.

3) Know what your readers anticipate and provide them with answers.

4) Challenge your peers about the severity of the research problem and put forward your proposal for a solution.

5) Finally, organize existing evidence relevant to the problem and interpret it clearly and logically.

As apparent from the above roadmap, develop your thesis statement (introduction) starting from a general argument and narrowing it to focus your rationale on how the research should proceed. The rest of your paragraphs in your introduction should support your claims. Don't argue on facts, but use them to prove or disprove your claims. Always give evidence from scholarly documents. In short, your statement is a fundamental part of your introduction. So, write your statement carefully and thoughtfully. After all, the main purpose of your introduction is to introduce your topic to the readers (Banus \& Dauda, 2015). Take note that readers may not read your introduction until the end if you fail to capture their attention and interest. Your introduction can only stand out if you relate it to everyday life and convince readers of its practical importance. Hence, your introduction must be motivating, current, and be based on a strong methodological and theoretical certainty.

\subsection{How to Write Your Problem Statement}

The problem statement states a specific condition (issue) that needs urgent attention and a possible solution. As a researcher, show your readers how your investigation will fill or narrow the gap in existing literature. An excellent problem statement is just a line or two. The rest of the paragraph(s) is its elaboration, discussing a possible solution and most importantly, why it is a problem that needs urgent attention (cite scholarly references). The problem must generate questions for the researcher to answer (Faryadi, 2012).

A $\mathrm{PhD}$ thesis problem statement must seek to answer the following questions:

1) What is the problem?

2) Where is the problem?

3) How can the problem be solved?

4) Why do you want to solve the problem?

5) Is the problem a current issue?

6) Will the problem persist if it is not solved?

7) Who are affected adversely by the problem?

8) Will this problem prove or disprove existing knowledge?

In short, the problem statement describes an existing issue which is so grave that it must be addressed. Generate questions about your problem statement and try to answer those questions to prove or disprove your research problem. Hence, the research problem is the main part of any scientific enquiry (Jacobs, 2013). 


\subsection{Importance of the Problem Statement}

1) It highlights the importance of your thesis topic.

2) It creates interest among researchers.

3) It adds new knowledge.

4) It identifies a gap in the literature.

5) It indicates a need for future research.

6) It stimulates thinking about what else is required to solve future related problems.

\subsection{Some Don'ts When Writing a Problem Statement}

1) Is it researchable? If your problem is so difficult or complex that it is beyond the ability of the researcher, then it will lead to a wastage of time and resources.

2) Do you have relevant scholarly documents? If nothing has been written about your research problem, perhaps the problem is not worthy of investigation.

3) Are you a suitable person to do this research? Are you qualified? Do you have the necessary skills to carry out this study? If your answer is no, then time and resources will go to waste if you proceed.

4) Will solving the problem bring practical benefits? Do you think this research contributes to society? Will it bridge the knowledge gap? Is your research challengeable? Which category of society will benefit from this research?

\subsection{Selection of a Research Problem}

1) Interest: Pose a problem that you find it fascinating so that you will be motivated to investigate it further.

2) Knowledge: Do a background study of your research problem so that you can discuss it with conviction and authority.

3) Conceptual frame work: Your research problem must be theoretically and conceptually sound.

4) Data availability: Investigate a problem that you can find materials and sources to support your investigation.

\section{How to Write Your Research Questions}

The research question is one of the essential parts of a thesis. It focuses on the study, regulates the methodology, and guides all the stages of investigation, analysis, and finally answers the problem statement. There is a difference between your topic and your research question. The topic is meaningless without a good research question. Your research question should reflect and elaborate a deeper understanding of your topic (Demir Kaçan, 2015). Your research question should also seek to erase all ambiguity about your research problem.

It may even guide you in the type of investigation you should conduct, identify the type of data you should collect, and lead you to a proper analytical approach of your data. In short, a good research question helps you to focus on fi- 
nishing your investigation without wasting much time and resources. Moreover, good research questions create a corridor to your research. They act even like the spine of your proposal and later, your thesis. Good questions are the ones that can be answered by researchers as they are supported by evidence. In short, good research questions that lead to investigations help eliminate serious problems from our society (Tenenberg, 2014).

\subsection{Writing Research Questions}

1) Choose a topic that interests you and your readers. It must be thought-provoking. Make an investigation of your topic by going through scholarly journals to see what questions are raised by your peers. Take note of what questions are not raised so that you can add them to your list. Your research question should not be answered just by simple facts; it should require critical analysis and field-tested research. Your research question should not cover an area that is too broad or too narrow. If it is too narrow, you will have difficulty finding relevant information.

2) Do not forget to show your research questions to your supervisor before going into details.

When formulating research questions, we ask how the research problem originated and subsequently progressed to a serious state. Researchers ask questions and put their hypotheses in context. The hypothesis indicates the researcher's line of thinking as he/she predicts specific relationships or situations. It may turn out to be true or false. As such, in order to hypothesize or make predictions, researchers need relevant knowledge and skills. To prove or disprove a hypothesis, researchers are compelled to ask questions about the problem to be studied.

They test the questions in the field by gathering relevant information and data to test their hypotheses, and by conceptual knowledge and thorough analytical investigation. When researchers carry out an investigation about a specific problem, they ask questions that require either a description or explanation. E.g. Does a skillful and knowledgeable person perform well in the field? Questions may also indicate what, where, and when a problem occurs. With explanatory questions, researchers always start with why. For instance: Why did the wall of that building collapse? What was the cause of it? Research questions can also indicate future events. What will be the final grade of your students once they use the new information system in their classroom? This type of question is a hypothetical or predicted question. It is all about state of the mind and understanding of the researcher. Prediction is not real knowledge. It becomes knowledge once it is investigated and proven. Beside descriptive or explanatory questions, researchers may also ask open-ended and closed-ended questions to solve research problems. A closed-ended question is just the opposite of an open-ended question.

A closed-ended question requires an answer that is either Yes or No. Did you attend your History class this evening? Answer: Yes/No. For an open-ended 
question, there is no specific response. It is open to discussion. For example: What do you think about my class performance this morning? You can see that the answer is not Yes or No, but it requires an opinion. Hence, as a researcher, you are required to ask various types of questions in order to obtain the necessary information for your study.

\subsection{Why Do Researchers Ask Question?}

1) Questions guide the researcher to find suitable answers to solve the research problem.

2) Asking questions helps the researcher focus on the topic under investigation.

3) It helps the researcher focus on the important issues in his/her thesis.

4) It helps the researcher find answers for his/her research hypotheses.

\section{What Is a Hypothesis?}

A hypothesis is an unproven reasoning. It enables an educated guess or a prediction of a result or relationship under specific conditions. It requires rigorous testing to determine its validity and reliability. Once it is proven after serious testing, it becomes a scientific theory. " $A$ hypothesis is an idea or explanation for something that is based on known facts but has not yet been proved." "A hypothesis (plural hypotheses) is a proposed explanation for a phenomenon." " $\mathrm{Hy}$ pothesis is an assumption or concession made for the sake of argument, an interpretation of a practical situation or condition taken as the ground for action, a tentative assumption made in order to draw out and test its logical or empirical consequences". Cambridge English Dictionary, Wikipedia, Merriam Webster Dictionary.

Please take note that although a hypothesis helps the researcher to see the problem more clearly, it is not necessarily a part of an investigation. If you have no hypothesis, you still have a valid research. But if you like to have hypotheses, you can construct as many as you wish. They are all just assumptions, right or wrong; you don't know until the end of your study. Your hypothesis is just an educated guess, declaration, an idea, assumption and feeling that you are not sure of. And that becomes a reason for your enquiry. As we have discussed above, a hypothesis is a reasoning that enables an educated prediction. To validate a research claim, we must test the hypothesis to determine whether it supports our claim or otherwise. Hypothesis testing is a very crucial procedure in research. When tested in the field, it will indicate whether our prediction is statistically significant or otherwise.

\subsection{Why Do We Need a Hypothesis?}

1) It helps to validate research activity.

2) The researcher can find out whether his assumption is true or false.

3) It directs the researcher in his quest for new knowledge.

4) A hypothesis helps save time and resources. 
5) It helps you collect and apply relevant data.

6) A carefully formulated hypothesis leads to a meaningful conclusion.

7) It helps you focus on specific issues and leads you to investigate what you are supposed to investigate.

8) It also assists you in the collection of relevant data.

9) A well supported hypothesis contributes to the validation of a theory and may even strengthen it.

\subsection{Common Types of Hypothesis}

Null Hypothesis (H0): This type of hypothesis indicates that whatever we hypothesize is believed to be correct or true. Example: There is no difference between students who use Facebook to learn English compared to students who do not use Facebook. So, there is no difference between the two groups of students.

Alternative Hypothesis (H1): This type of hypothesis indicates that whatever we hypothesize is believed to be not correct or not true. Example: There is a difference between students who use Facebook to learn English compared to students who do not use Facebook. So, there is a difference between the two groups of students.

\subsection{How Do You Test Your Hypothesis?}

1) Choose the type of hypothesis you want to test.

2) Determine the sample size of the selected population to test.

3) Collect your data.

4) Determine the significance level.

5) Compare your P-value to see the significance level.

6) Make a decision whether to accept or reject your hypothesis.

It is clear, therefore, that the research question and the hypothesis are the spine of any thesis writing process. These two components will determine the success or failure of any research. Without research questions and hypothesis, the research aim, purpose, and problem cannot be established clearly. Hence it is vital that your hypothesis and research question are well-constructed. Your hypothesis must be simple, relevant to your study, testable in the field, verifiable, and most importantly realistic. If it lacks the above characteristics, the outcomes will not be desirable.

\subsection{Errors in Hypothesis}

Once you put your hypothesis to the test there will be two outcomes:

1) Correct.

2) Incorrect.

It is possible to make mistakes in our judgement when testing a hypothesis even though the result is correct. This happens due to the following:

1) Your research road map is not correctly designed.

2) Your population or sampling is not adequate or suitable. 
3) Your data analysis and statistical approach are questionable.

4) Your conclusion is not correct.

There are two types of errors:

1) Type 1 Error: It happens when you reject a null hypothesis that is indeed true.

2) Type II Error: It happens when you accept a null hypothesis that is indeed false.

\section{Contribution of Your Research}

Since a $\mathrm{PhD}$ research is a scientific discovery of new knowledge, other researchers in the related field will benefit from your findings. Your thesis will narrow or bridge the gap in the literature with regard to the topic of your research. Others will be able to apply your findings in their attempt to solve a similar problem. As such, your investigation and findings must be genuine and original. Outline a list of contributions that you think your findings add to the literature. Your contributions must be specific and focused. Provide significant evidence indicating that your research is worthy of investigation. The following are some examples of how a $\mathrm{PhD}$ research can make useful contributions. Contribution to knowledge means creating new knowledge based on the previous available knowledge by doing extensive and innovative research in order to prove something right or wrong. The Most Important issue in research contribution is the "Significance." The problem is: the word significance is subjective. It is significant to you but may be not significant to me. Here you are! So, the researcher has to measure the contribution not to claim only. There are seven ways to measure significance so that you present your contribution scientifically:

1) Explain the importance of your research problem.

2) Your findings.

3) Transformation of the existing theory.

4) Explain generalizability or lake of generalizability, your added values: Economic value? Cultural value? Societal value, scope and scale of your findings and its application. I am sure, all scholars agree that Size does not matter in research contribution ... so show me the evidence where, how.

5) Experimental significance. You experimented it in the field not just talking theoretically. So, you confirmed a new knowledge scientifically. What is the new knowledge? You bridged the gap, you answered your problem statement, research questions and hypotheses. So, now based on your problem can specifically mention the new knowledge.

6) Your vital significance is Your Research Area.

7) Another vital significance is your research method. The ways you conducted your research and it worked. Your interviews, questionnaires, data collections, the way you analysed your data and your literature search, all of these methods are important contributions of your research. Your creativity in solving your problem and answering your hypothesis are significant contri- 
butions to future researchers. As thus your method of solving a problem creatively using different approach adds an important impact to the body of existing knowledge.

For example: my problem statement: "Magnesium chloride helps to reduce joint pain among Malaysian senior citizens." So, you did your experimental on the Malaysian senior citizens and found that it really does. Magnesium chloride really reduced or eliminated their joint pains. Obviously, you invented, discovered a solution and bridged the gap. What is the gap? The joint pain among Malaysian senior citizens that we did not know previously and now we know. You see? This is how you measure significance with scientific evidence.

\subsection{What Is So Called "Body of Knowledge?}

It refers to a complete system whereby all professionals, scholars and scientists contribute collectively their expertise and skills. As such, it demands that any new professionals who would like to be part of this system must contribute new knowledge into the existing system in order to be recognized as a member of that professional system (body of knowledge). Have you heard about the contribution of a PhD thesis to the body of knowledge? The author of the $\mathrm{PhD}$ thesis must contribute a new skill, new knowledge into the existing system so that he/she can be recognized as a professional member of the body of knowledge. That's why it is called "Body of Knowledge" or (B.O.K).

\subsection{Examples That You Strive to Prove Contribution}

There is not enough investigation and scientific research conducted about your topic of investigation. May be written but not empirically documented. Or even empirically documented but the findings are in dispute. So, you want to challenge the findings and would like to prove or disprove the original research. $\mathrm{Re}$ member, if your research to be scientifically valid, it must be verifiable, re-do-able and challengeable by other researchers. How original is your research? Is it Current? Only you did it? Is the whole idea yours? Or you are expanding an existing idea? What advancement you brought to our knowledge? Did you Prove or disprove a knowledge? What is the impact of your research? Where you did the impact? Did you confirm and Verified your research? If your research to be valid, it must be verifiable, re-do-able, challengeable and so on. Only then you have an impact and a contribution.

\section{Conclusion and Implication}

This paper discussed the $\mathrm{PhD}$ thesis writing process. As evident from the above discussion, your thesis should be a scientific and scholarly piece of work, having fulfilled all the above stipulated scientific conditions. Your PhD thesis is not a simple book, but a scientific document. It contains scholarly work, researched material, concepts, experimentations, contributions and findings that could solve or mitigate a problem. A $\mathrm{PhD}$ thesis has a pre-determined and globally 
standardized format and outline. It begins with a brief proposal indicating the What, Why, How, and When of your thesis. Your proposal is the first stage, and it is crucial to state it correctly.

Teachers, learners and researchers, even educational policy makers, will find this paper as a vital reference point and a useful guide. The prime objective of this paper is to outline a suitable road map for $\mathrm{PhD}$ candidates, researchers and scholars about the how, when and why of doing scientific research. This paper helps researchers to systematically design, plan and effectively implement a viable course of action in their investigation. As such, the time has come to replace the existing boring research methods with the one that is easy, step by step and scientifically sound. When a right methodology of research is used in the field, research investigation is facilitated. Hence, as researchers, we need to think critically as well as creatively so that we can identify effective and correct methodology of research.

\section{Conflicts of Interest}

The authors declare no conflicts of interest regarding the publication of this paper.

\section{References}

Al-Raqqad, H. K., Al-Bourini, E. S., Al Talahin, F. M., \& Aranki, R. M. E. (2017). The Impact of School Bullying on Students' Academic Achievement from Teachers Point of View. International Education Studies, 10, 44-50. https://doi.org/10.5539/ies.v10n6p44

Banus, A. A., \& Dauda, B. (2015). Effects of Understanding the Problem Statement on Students' Mathematical Performance of Senior Secondary Schools in Borno State, Nigeria. Journal of Education and Practice, 6, 6-11.

Demir Kaçan, S. (2015). Examining Research Questions on Germination from the Perspective of Scientific Creativity. Journal of Education and Practice, 6, 119-121.

Faryadi, Q. (2012). How to Write Your PhD Proposal: A Step-By-Step Guide. American International Journal of Contemporary Research, 2, No. 4.

Faryadi, Q. (2017). Everything You Need to Know about. PhD Thesis Writing from Proposal to VIVA. Kuala Lumpur: Future Expert Solutions.

García, S., \& Fombona, J. (2015). Approach to the Phenomenon of M-Learning in English Teaching. Digital Education Review, 28, 19-36.

Jacobs, R. L. (2013). Developing a Dissertation Research Problem: A Guide for Doctoral Students in Human Resource Development and Adult Education. New Horizons in Adult Education \& Human Resource Development, 25, 103-117. https://doi.org/10.1002/nha3.20034

Kafes, H. (2018). A Genre Analysis of English and Turkish Research Article Introductions. Research on Youth and Language, 12, 66-79.

Koçyigit, M., \& Erdem, C. (2018). A Content Analysis of Graduate Research on English Preparatory Programs at Universities. International Journal of Human Sciences, 15, 1466-1480. https://doi.org/10.14687/jhs.v15i3.5414

Mincu, M. E. (2015). Teacher Quality and School Improvement: What Is the Role of Research? Oxford Review of Education, 41, 253-269.

Tenenberg, J. (2014). Asking Research Questions: Theoretical Presuppositions. ACM Transactions on Computing Education, 14, Article 16. https://doi.org/10.1145/2644924 